\title{
Workshop report: 4th European crystal network meeting
}

\author{
Frédéric Lioté, Tony Merriman, Sonia Nasi and Alexander So*
}

The 4th workshop of the European Crystal Network took place recently in Paris. Approximately 40 biomedical scientists took part in this closed meeting, whose aim was to facilitate scientific exchange and foster collaborations between centers involved in research in crystal-induced inflammation and crystal deposition diseases. Rheumatology was well represented at the meeting, but there were also participants from other medical disciplines (genetics, epidemiology, nephrology) and basic scientists with diverse interests (crystallography, biochemistry, inflammation, physics).

Within the rheumatic diseases, a major focus is gout and hyperuricemia, but the role of calcium crystals in disease is also gaining interest. Recent advances in genetics, inflammation research and the biochemical pathways that mediate calcium crystal deposition were some of the highlights of this year's meeting. New clinical and therapeutic aspects of hyperuricemia and gout were also discussed. This report summarizes the subjects that were presented with reference to gout and hyperuricemia. Other presentations on the mechanisms of tissue calcification and their possible role in osteoarthritis are not summarized here.

\section{Hyperuricemia and gout}

What is the normal serum uric acid level?

Thomas Bardin (Paris, France) reviewed the current definition of what the normal level of serum uric acid (SUA) is based on. The current definition is population based and is remarkably similar to the solubility threshold of $420 \mu \mathrm{mol} / \mathrm{l}(7.0 \mathrm{mg} / \mathrm{dl})$. Interestingly, this SUA level also predicted incident gout in epidemiological studies. However, up to $10 \%$ of gout patients had SUA levels between 6.0 and $7.0 \mathrm{mg} / \mathrm{dl}$, suggesting that monosodium urate (MSU) crystals can also form at lower concentrations. He suggested that $6.0 \mathrm{mg} / \mathrm{dl}$ may be a more appropriate normal SUA concentration, and

\footnotetext{
* Correspondence: Alexanderkai-lik.so@chuv.ch

Service de Rhumatologie, Centre Hospitalier Universitaire Vaudois, Ave Pierre Decker 4, Lausanne 1011, Switzerland
}

this value is also the recommended target level for urate-lowering therapy. In gout cases there is no reason to have different normal SUA levels between men and women, because the phenomenon of crystal formation is the same.

\section{Proteomic analysis in intercritical gout}

Tim Radstake (Nijmegen, the Netherlands) presented data from ongoing studies on the proteomic analyses of serum from patients in the Netherlands and New Zealand with acute and intercritical gout. These studies found that IL-8 levels were significantly increased in both situations. A different proteomic profile, which correlated with S100A8 levels, was observed when patients with high or low IL-8 levels were compared.

\section{Imaging in the diagnosis of gout}

Nicola Dalbeth (Auckland, New Zealand) presented a review on current imaging techniques in gout. Dualenergy computed tomography scan provided a clear demonstration of MSU deposits around joints and tendons. Future research should focus on comparison between the different imaging modalities (ultrasonography study and dual-energy computed tomography) in early gout or even in asymptomatic hyperuricemia.

\section{Urate excretion in the general population}

Muriel Bochud (Lausanne, Switzerland) reported data on urinary fractional excretion of uric acid (FEUA) from COLAUS, a population-based cohort that included 6,000 Caucasian participants, aged 35 to 75 years, in Lausanne. SUA as well as urinary urate from spot urine samples were measured in each subject. The results showed that FEUA is higher in women than in men. There is a clear negative independent association of FEUA with body mass index, mainly fat mass, fasting insulin levels, triglyceride levels, $\gamma$-glutamyltranspeptidase levels, estimated glomerular filtration rate (Chronic Kidney Disease Epidemiology Collaboration equation), and ultrasensitive C-reactive protein. These preliminary results highlight 
that metabolic factors are associated with FEUA in adults from the general population.

\section{Fructose loading and urate handling}

Tony Merriman (Dunedin, New Zealand) reported the results of physiological overload of oral fructose loading. Epidemiological data suggest a relationship between sugar-sweetened soft drink consumption and gout. In collaboration with Nicola Dalbeth, he studied whether genetic variation in SLC2A9, a fructose and urate transporter, influences the acute urate response to a fructose load. Caucasians without the protective $\mathrm{C}$ allele had a higher increase in serum urate and lower FEUA. No effect was seen in people of Polynesian ancestry. They concluded that SLC2A9 might have a greater influence on the renal response to sugary beverages in Europeans. Additional results were provided on the effects of a genetic variant for ABCG2, another tubule efflux transporter. Their data showed that the urate-raising variant of ABCG2 increased FEUA.

\section{Tophus and bone destruction}

From the literature and from her own pathological studies, Nicola Dalbeth (Auckland, New Zealand) described the pathology of a tophus. MSU crystals are surrounded by a cellular corona of inflammatory and resident macrophages, including multinucleated giant cells that have features of osteoclasts. Indeed, in vitro experiments demonstrated direct stimulatory effects of MSU crystals on osteoclasts, and, by contrast, inhibitory effects on osteoblasts.

\section{Adrenocorticotropic hormone as a treatment of acute gout}

Fernando Perez-Ruiz (Vizcaya, Spain) reported on the efficacy of adrenocorticotropic hormone as a treatment for gout flares based on open and short-term studies. The anti-inflammatory effects of adrenocorticotropic hormone might rely on steroid effects and on peripheral action through activation of the melanocortin type 3 receptor expressed on monocyte-macrophages (via inhibition of cell migration mediated by cytokine and adhesion molecule expression and the ability to interfere with cell signaling pathways). Current randomized trials comparing adrenocorticotropic hormone and steroid depot injection in patients with acute gout are ongoing in Spain.

\section{Cellular effects of crystals}

\section{$\mathrm{P} 2 \mathrm{X7}$ receptor and inflammasome activation}

Francesco Di Virgilio (Ferrara, Italy) reviewed the biology of the P2X7 receptor. The P2X7 receptor binds extracellular ATP and is a potent activator of the NLRP1 and NLRP3 inflammasomes. Potential mechanisms include changes in the intracellular $\mathrm{K}^{+}$and $\mathrm{Ca}^{2+}$ concentrations. Pathogenassociated molecular patterns and danger-associated molecular patterns were also shown to interact directly with scaffold proteins of the inflammasomes and initiate their activation. Finally, a body of evidence shows that the inflammasomes may be involved in the regulation of cell growth and differentiation.

\section{Signal transduction in basic calcium phosphate crystal- induced inflammation}

Geraldine McCarthy (Dublin, Ireland) reviewed the pathologies associated with basic calcium phosphate deposition. In vitro, basic calcium phosphate promotes cytokine and matrix metalloproteinase production and drives IL-1 $\beta$ and IL-18 production following NLRP3 inflammasome activation. Dr McCarthy demonstrated a role for Syk and phosphoinositide-3 kinases and characterized events downstream of Syk activation to identify novel targets for treatment of basic calcium phosphaterelated arthritis. Basic calcium phosphate also induced S100A8 release, and led to the expression of pro-IL-1 $\beta$ and pro-IL-18. These results suggest that Syk may be a potential therapeutic target in basic calcium phosphaterelated disorders.

\section{a-antitrypsin in gout}

Leo Joosten (Nijmegen, the Netherlands) discussed inflammasome-independent mechanisms of IL-1 $\beta$ by MSU crystals in mice and showed that neutrophil serine proteinases such as PR3 were implicated. $\alpha 1$-antitrypsin (AAT) is an abundant serine proteinase inhibitor and is able to inhibit PR3. The effect on MSU-induced inflammation was explored using human AAT-transgenic mice and a recombinant AAT-Fc fusion protein. AATtransgenic mice were protected against joint inflammation following intra-articular injection of MSU with free fatty acids, and treatment with AAT-Fc fusion protein suppressed joint swelling and local cytokine production as effectively as a high dose of IL-1R $\alpha$ (10 mg/mouse). AAT-Fc treatment was effective up to 48 hours before induction of gouty arthritis, indicating a long half-life for this compound. These data indicate that AAT-Fc may be a novel anti-inflammatory therapy for patients suffering from gout.

\section{Genetics}

\section{Genetics of serum urate}

Anne Köttgen (Freiburg, Germany) presented 18 new genetic variants associated with serum urate levels in a genome-wide association scan performed by the Global Urate Genetics Consortium involving a meta-analysis of 48 studies ( $>110,000$ European subjects). These variants also increased gout risk. The new genetic variants clustered into glycolysis and inhibin-activin signaling 
pathways and, significantly, no new uric acid transporters were discovered to add to the previously identified transporters (for example, SLC2A9 and ABCG2). Dr Köttgen hypothesized that the glycolysis genes could increase lactate levels and influence renal excretion of uric acid. How the inhibin-activin genes could influence urate levels is not clear because they encompass a wide range of molecular pathways.

\section{Heritability of gout}

Dr Chang-Fu Kuo (Nottingham, UK) described the use of the National Health Insurance Research Database of Taiwan to estimate heritability of gout. This database includes demographic and phenotypic information from $>98 \%$ of the Taiwanese population (23.03 million individuals in 2010) and genealogical data, from which 16.8 million primary relative pairs were identified. Gout was defined using the ICD9 code and treatment associated with gout, with the overall prevalence of gout being $4.62 \%$ ( $7.07 \%$ in males and $2.15 \%$ in females). Heritability was estimated under a polygenic model with environmental effects estimated using spouses. The estimated heritability of gout was $33 \%$ in males and $17 \%$ in females. Dr Kuo pointed out that the model used would provide a conservative estimate of heritability compared with twin studies.

\section{Relationship between urate and renal function}

Tony Merriman (Dunedin, New Zealand) presented data on increased serum urate predicting poor renal function independent of other measured risk factors. However, whether urate per se is detrimental to renal function remains unresolved. The use of Mendelian randomization that uses genetic variants to test for a causal role of urate on renal function was described. Data from the Atherosclerosis Risk in Communities and Framingham Heart studies were used and a uric acid transporter genetic risk score was employed as the instrumental variable to test for a causal role of urate in renal function. Overall, an increased genetic risk score was associated with improved renal function in men but not in women.

\section{Genetic determinants of urate excretion}

Murielle Bochud (Lausanne, Switzerland) described the SKIPOGH study, which aims to disentangle the genetic determinants contributing to renal and hypertensive phenotypes. This study contains 856 parent-offspring pairs and 414 sibling-sibling pairs, all of which have comprehensive renal phenotype data. Heritability estimates of serum urate, FEUA, 24-hour uric acid clearance and urinary urate clearance were $0.42,0.45,0.43$ and 0.17 , respectively. Good phenotype ascertainment is very important in this kind of study; for example, in a Seychelles study the heritability of blood pressure was estimated as $\sim 10 \%$ based on one measure, as $\sim 20 \%$ based on five measures and as $\sim 40 \%$ based on 10 measures.

\section{Role of SLC2A9 gene expression in hyperuricemia and gout}

Philip Riches (Edinburgh, UK) reviewed the currently understood role of the renal uric acid transporter SLC2A9 (GLUT9) in the proximal renal tubule. Genetic variants in SLC2A9 are strongly associated with serum urate levels, explaining $3.5 \%$ of phenotypic variance. SLC2A9 exists as two isoforms - a full length isoform, and an isoform missing the $\mathrm{N}$-terminal 28 residues. Expression of the short form (on the apical membrane) correlates with the urate level, with the full-length isoform on the basolateral membrane acting as a dominant mediator of uric acid exchange. Dr Riches presented data showing differential renal expression of distinct SLC2A9 genetic variants, with evidence for an internal promoter. SLC2A9 is also expressed in synovial tissue.

\section{Eurogout}

Eurogout is a European Gout Genetics Consortium, established under the aegis of the European Crystal Network in 2010 for the recruitment of a very large, wellascertained gout sample set for use in genome-wide genetic studies. Progress was updated $-1,171$ cases have been recruited.

\section{Abbreviations \\ AAT: a1-antitrypsin; FEUA: Fractional excretion of uric acid; IL: Interleukin; MSU: Monosodium urate; SUA: Serum uric acid.}

\section{Competing interests}

The authors declare that they have no competing interests.

\section{Acknowledgements}

The meeting was supported by unrestricted grants from the following pharmaceutical companies - Menarini France, Savient and SOBI. Meeting organizers: Alexander So and Frédéric Lioté.

Published: 25 Oct 2013

10.1186/ar4348

Cite this article as: Lioté et al:: Workshop report: 4th European crystal network meeting. Arthritis Research \& Therapy 2013, 15:304 